\title{
The Mechanism of Lipofectamine 2000 Mediated Transmembrane Gene Delivery
}

\author{
Shaohui Cui, Shubiao Zhang, Huiying Chen, Bing Wang, Yinan Zhao, Defu Zhi \\ Key Laboratory of Bio-chemistry Engineering - The State Ethnic Affairs Commission-Ministry of Education, \\ Dalian Nationalities University, Dalian, China \\ Email: csh@dlnu.edu.cn
}

Received 2012

\begin{abstract}
In this paper, the relatived mechanism between lipofectamine 2000 mediated transmembrane gene delivery and endocytic pathway were investigated. Clathrin and caveolae-mediated endocytic pathway contributions to transfection efficiency were studied. The inhibitors of endocytosis were used to treat HEp-2 cells before lipofectamine 2000/pGFP-N2 transfection. Transfection efficiency was evaluated with green fluorescence protein (GFP) expression assays. Cell viability and cytotoxicity were evaluated with MTT method. The results indicated that inhibitors of clathrin (chlorpromazine or wortmannin) and caveolin (genistein) could reduce the cell transfection efficiency observably. Both clathrin and caveolae-mediated endocytic pathways play important roles in transmembrane gene delivery.
\end{abstract}

Keywords: Gene Delivery; Cationic Liposomes; Transfection Efficiency; Endocytic Pathway; Inhibitor

\section{Introduction}

Non-viral DNA delivery systems have been developed to facilitate gene entry into mammalian cells ${ }^{[1]}$. It has shown that non-viral vectors have the advantages over viral ones as they are non-immunogenic, easy to produce and not oncogenic. However, they have the major limitation of inefficient transfection $^{[2]}$. Indeed, non-viral vectors showing much lower toxicity to cell proliferation were commercially available for lipoplexes mediated transfection (lipofection) of cultured cells. However, despite several studies investigating the mechanisms of uptake and intracellular trafficking of non-viral vectors to improve delivery, the current understanding of these processes was still limited. Because cell membrane was the first barrier for lipoplexes entry into the cell, the mechanism of cationic liposomes mediated transmembrane gene delivery was important as the study could contribute to improving transfection efficiency. There is convincing evidence that endocytosis represents the major pathway of lipoplexes entry into cells that leads to productive gene expression, rather than that genes are translocated into the cytosol at the level of the plasma membrane ${ }^{[3-4]}$. However, there are various endocytic pathways operated in eukaryotic cells, such as clathrin-dependent and clathrin-independent pathways, the latter includes phagocytosis, macropinocytosis and caveolae-mediated internalization ${ }^{[5]}$. The relative contribution of each pathway in lipoplexes internalization has been poorly defined to date, although involvement of the clathrin-mediated pathway has been firmly established, while evidence was emerging that entry may also occur via macropinocytosis $^{[4-6]}$. Endocytotic inhibitors such as chloroquine, chlorpromazine are very helpful for studying drug intracellular release or elucidating a specific endocytosis route ${ }^{[7]}$. There are still many problems that have not been elucidated on the biological barriers and properties of gene delivery through non- viral vectors, although much effort has been expended on them. Understanding the mechanism of transmembrane might play a significant role in overcoming the hurdles in gene delivery. This study was performed with cationic liposome, lipofectamine 2000 which showed higher transfection efficiency. The inhibitors of clathrin and caveolin, such as chlorpromazine, wortmannin and genistein were used to treat with cells. Via evaluated inhibitory effects of lipoplexes transfection to explore the underlying transmembrane mechanism in HEp-2 cells.

\section{Experimental}

\subsection{Materials and Equipment}

Human throat epidermis (HEp-2) cancer cell line was purchased from Cell Bank of Chinese Academy of Sciences. RPMI-1640, DMEM, Fetal bovine serum (FBS) were purchased from HyClone. Lipofectamine 2000 transfection reagent were purchased from Invitrogen Corporation. Plasmid pGFP-N2 (containing green fluorescent protein gene) was purchased from Clontech Company. MTT, Wortmannin, Chlorpromazine, Genistein were purchased from sigma-Aldrich.

Inverted fluorescence microscope (Olympus IX 71) was purchased from Japan. Microplate reader (Sunrise Tecan) was purchased from Australia. Carbon dioxide incubator (NAPCO 7100) was purchased from France.

\subsection{Cell Culture}

HEp-2 cells were grown in $100 \mathrm{ml}$ culture flask in RPMI-1640 supplemented with $10 \%$ fetal bovine and antibiotics (100 U penicillin/ml and $100 \mathrm{mg}$ streptomycin/ml) at $37^{\circ} \mathrm{C}$ in $5 \% \mathrm{CO}_{2}$ incubator.

\subsection{Lipoplexes Preparation}


Preparation of lipoplexes (50 $\mu \mathrm{l} /$ well amount of calculation): The plasmid DNA (pGFP-N2) $0.2 \mu \mathrm{g}$ and transfection reagents Lipofectamine 2000 were diluted in Opti-MEM 25 $\mu$, and then the diluted transfection reagent dropping $25 \mu \mathrm{l}$ dilutions of plasmid DNA to $25 \mu \mathrm{l}$, the side with the vortex oscillator plus side, at room temperature and incubated for $20 \mathrm{~min}$. The weight ratio of lipofectamine 2000 to pDNA was kept constant at 3:1.

\subsection{Transfection Procedure}

HEp-2 cells $\left(1 \times 10^{5}\right.$ cells $\left./ \mathrm{ml}\right)$ were seeded into 96-well plates in $100 \mu \mathrm{l}$ growth medium (RPMI-1640) until the required cell number was obtained (80\% confluence) at the time of transfection. Cells were washed once with PBS and 50 $\mu$ l transfection lipoplexes (prepared as described above) were added to each well. It was mixed gently by rocking the plate back and forth. The cells were incubated in DMEM (not containing FBS) under $5 \% \mathrm{CO}_{2}$ at $37^{\circ} \mathrm{C}$ in a humidified incubator for $4-6 \mathrm{~h}$, then cells were washed by PBS once and medium was replaced by complete medium and cells were further cultured for $48 \mathrm{~h}$, prior to analysis. Finally, assay for transgene expression or selection for stable transfection was done according to report gene.

\subsection{Green Fluorescence Protein (GFP) Assay}

The expression of green fluorescence protein was imaged by inverted fluorescence microscope and transfection efficiency was given as relative efficiency. The transfection efficiency was determined based on cell percent with GFP-expressing. The number of GFP-expressing cells versus the total cell quantity in the microscope was defined as the transfection efficiency. Cell counting was performed randomly in microscopic observation scope under $10 \times 16$ magnifications with three repeats. Data were presented as the mean \pm standard deviation (SD).

\subsection{Cytotoxicity Assay}
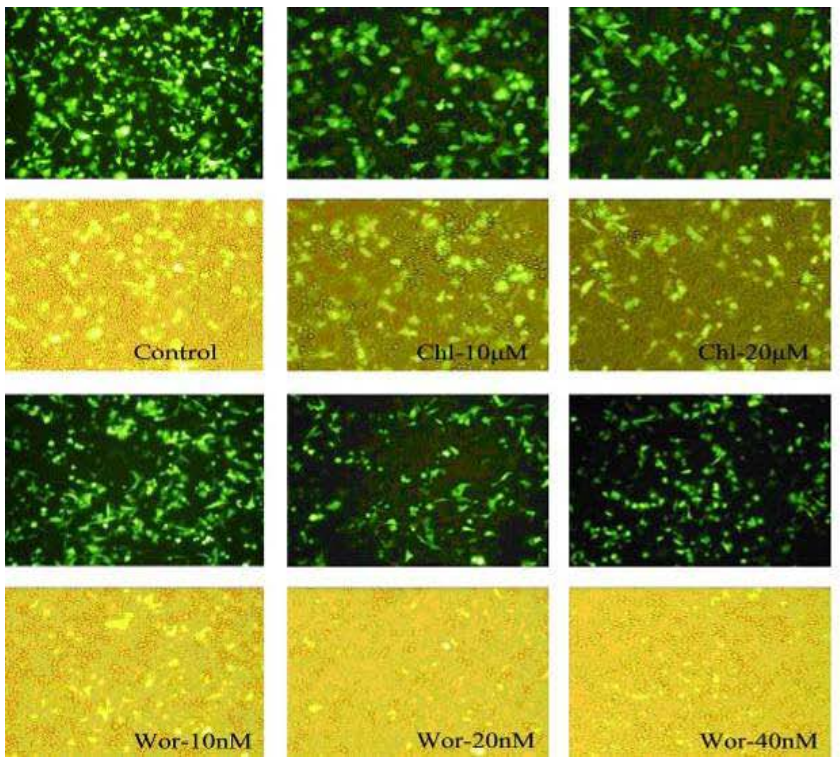

The cytotoxicity was evaluated by MTT assay. Hep-2 cells $\left(1 \times 10^{5}\right.$ cells $\left./ \mathrm{ml}\right)$ were seeded into 96 -well plates at $200 \mu \mathrm{l} /$ well and incubated $24 \mathrm{~h}$ after. $50 \mu \mathrm{l}$ MTT $(5 \mathrm{mg} / \mathrm{ml}$ in PBS) was added and incubated for an additional $4 \mathrm{~h}$ at $37^{\circ} \mathrm{C}$. MTT-containing medium was aspirated off and $150 \mu \mathrm{DMSO}$ was added to dissolve the formazan and the absorbance was measured at $570 \mathrm{~nm}$ to determine cell viability as percentage of control. Non-treated cells as control.

\section{Results}

\subsection{Inhibitors of Clathrin Influenced Transfection Efficiency of Lipoplexes}

Endocytosis is the process by which cells take up macro- molecules from the surrounding medium. The best character- ized process is the so-called clathrin-dependent endocytosis. To assess whether clathrin-mediated endocytosis influenced transfection efficiency two inhibitors of clathrin (chlorprom-azine and wortmannin) were used to treated HEp-2 cells 1 hour before transfection. The transfection efficiency of lipofectamine2000/pGFP-N2 complexes was evaluated by GFP expression which showed significantly higher inhibition than lipofectamine2000 control. Figure 1 showed the represent-ative fluorescence profiling of positive GFP-expressing cells and whole cell contrast. Chlorpromazine $(10-100 \mu \mathrm{M})$ could observably lower transfection efficiency 1-12-fold (Figure 1 a, b) and wortmannin (10-100nM) had 0.8-15-fold (Fig. 1 c, d) compared with control. These results suggested that both chlorpromazine and wortmannin could lower transfection efficiency through inhibiting clathrin pathway. Endocytosis is also currently known about clathrin-independent endocytic processes such as those involving caveolae and lipid rafts. An understanding of endocytosis and the cellular trafficking that occurs thereafter has a great deal of relevance to current molecular medicine.
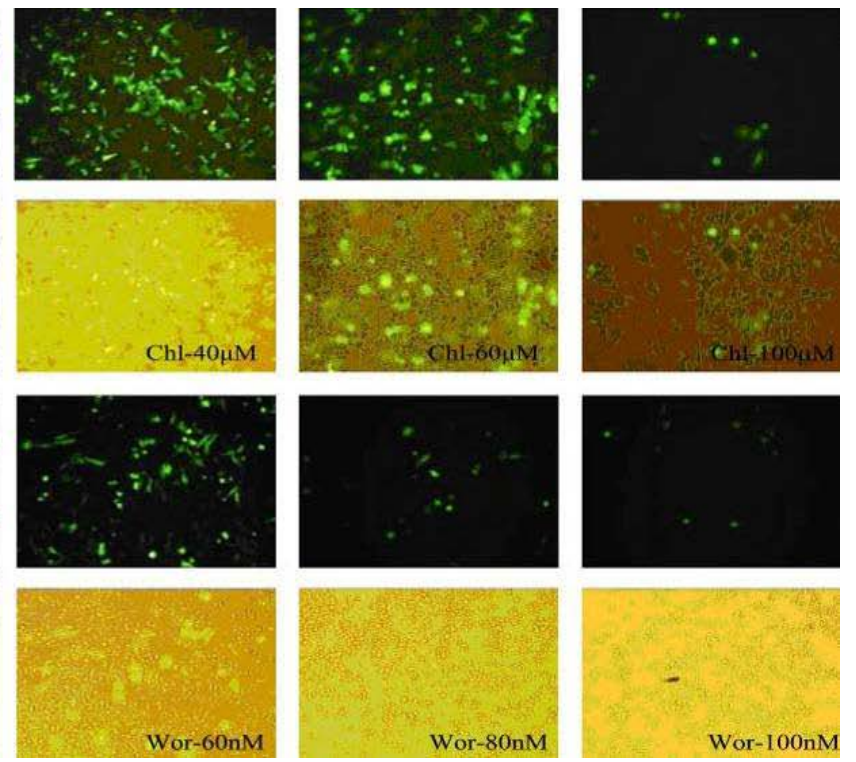

Figure 1. Effects of treatment with different concentrations of chlorpromazine $(10 \sim 100 \mu \mathrm{M})$ and wortmannin $(10 \sim 100 \mathrm{nM})$ on $\mathrm{HEp}-2 \mathrm{cells}$ 1 hour after transfected lipofectamine 2000/pGFP-N2 complexes experimental image ( inverted fluorescent microscope photos:20×10) contrast living cell. 
In addition, the cytotoxicity was tested with chlorproma-zine and wortmannin by MTT assay which could influenced cell viability and the valuation of cell transfection efficiency. Cell viability were $85 \%, 80 \%, 74 \%, 70 \%, 65 \%$, 60\% respectively when treated cell with chlorpromazine $(10,20,40,60,80$, $100 \mu \mathrm{M}$ ) (Figure not showed). The cytotoxicity of chlorpromazine was lower. The cytotoxicity of wortmannin was tested from $10-100 \mathrm{nM}$. When the concentrations of wortmannin exceeded $60 \mathrm{nM}$, cell survival rate was under 50\% (Figure 2). Therefore, the cytotoxicity could influence to evaluate transfection efficiency seriously. But, between $10 \mathrm{nM}$ and $60 \mathrm{nM}$, wortmannin could inhibite the transfection efficiency.

\subsection{The Effects of Inhibitors of Caveolin on Lipoplexes Cell Transfection}

In order to explore caveolae-dependent internalization pathway

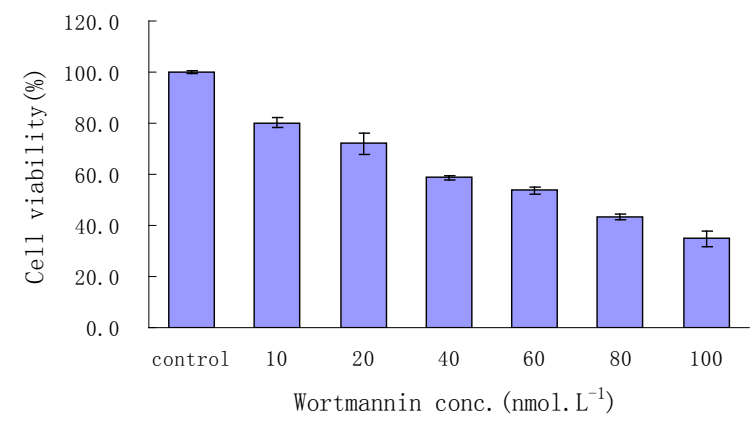

effects on transfection processes. Genistein, the inhibitor of caveolin, was used to treat HEp-2 cells 1 hour before cell transfection. Caveolins are essential for the formation and stability of caveolae. Figure 4 showed (part of pictures) the transfection efficiency was reduced $80 \%, 73 \%, 65 \%$, 45\% with genistein $(10,50,100,200 \mu \mathrm{M})$, respetivley, in terms of GFP expression than control. These results demonstrated the mechanisms of transmembrane transport of lipoplexes by caveolae-dependent pathway into the cell membrane channels. Through inhibiting the activities of caveolin Genistein could reduce caveolae-mediated endocytosis and transfection efficiency compared with Lipofecamine 2000 control. The cytotoxicity of genistein $(10-100 \mu \mathrm{M})$ was very small with the cell viability of above $80 \%$, but genistein with the concentrations over $100 \mu \mathrm{M}$ could cause cell viability to lower much (Figure 3).

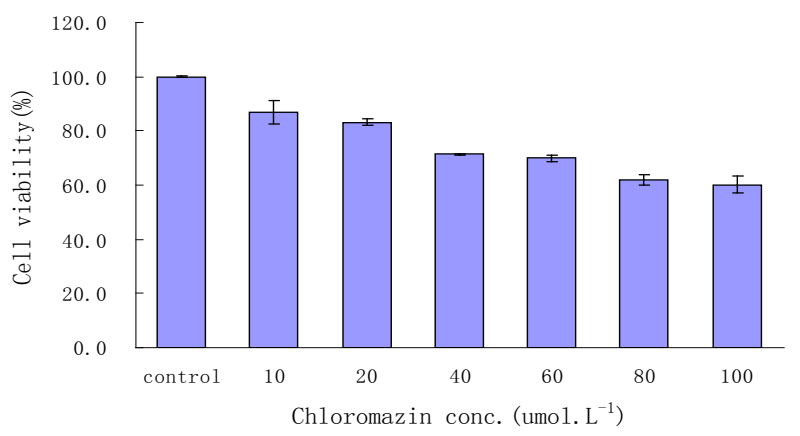

Figure 2. HEp-2 cells treated with different concentrations of inhibitor : wortmannin and chlorpromazine1hr, then was determined with the tetrazolium-based colorimetric cell viability. Non-treated cells as control. Error bars show standard deviation $(n=4)$.

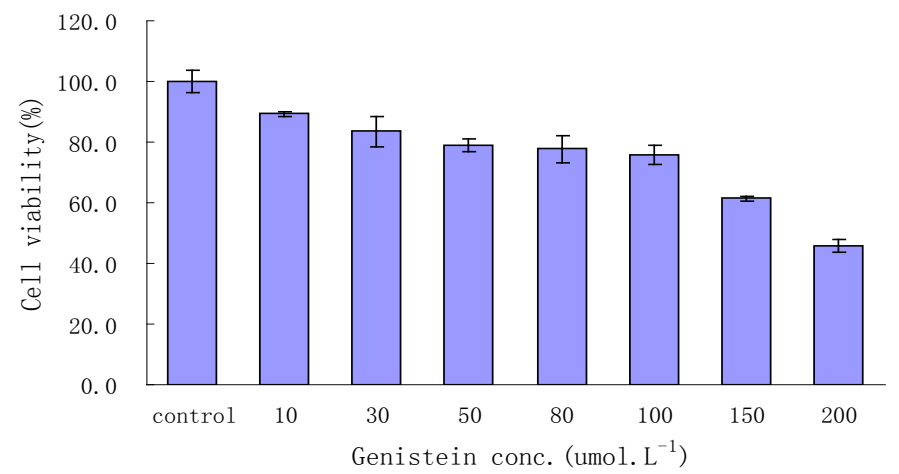

Figure 3. HEp-2 cells treated with different concentrations of genistein $1 \mathrm{hr}$, then was determined with the tetrazolium-based colorimetric cell viability. Non-treated cells as control. Error bars show standard deviation $(n=4)$.
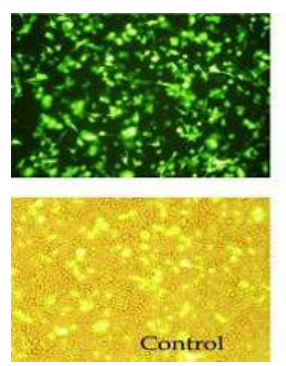
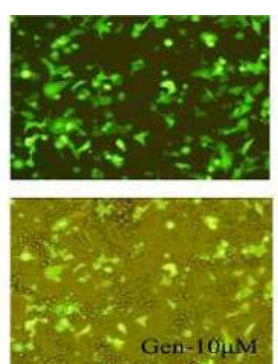
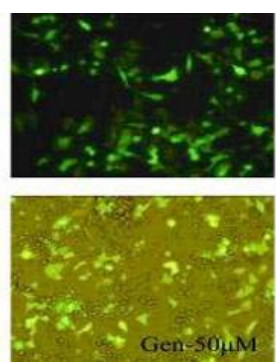
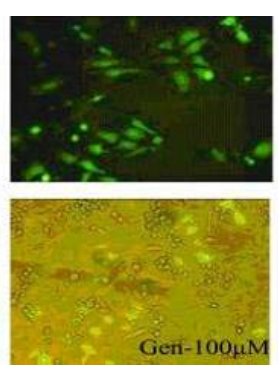
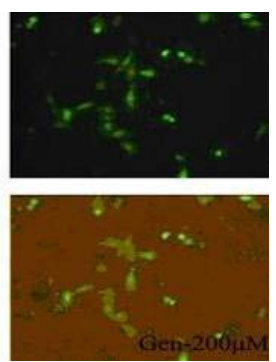

Figure 4. Effects of different concentrations of genistein on HEp-2 cells transfected Lipofectamine 2000/pGFP-N2 complexes experimental image ( inverted fluorescent microscope photos: $20 \times 10$ ). 


\section{Discussion}

Knowledge about the transmembrane uptake mechanism is important for the development of more efficient carriers. Cell membrane was the first barrier for internalization of lipoplexes. Some results indicated that endocytosis was the major mechanism of entry. Lipoplexes via which endocytic pathways entry into cell is of great importance for the design of further improved non-viral gene delivery systems. There are various endocytic pathways in eukaryotic cells ${ }^{[8-9]}$. Therefore, the aim of this study was to investigate the endocytic pathways were involved in cationic liposome mediated transmembrane gene delivery. A correlation between clathrin-mediated or Caveolae-dependent endocytosis and lipoplexes transfection was supported convincingly by several pieces of evidence, including the use of inhibitors of endocytosis, co-localization with pathway-specific markers and defective in clathrin-mediated endocytosis. They failed to internalize lipoplexes and concomitantly showed a proportional decrease in transfection efficiency. A combined approach was essential, since the use of inhibitors alone might give rise to unambiguous outcome because of toxic side effects. Our research focused on plasma membrane endocytic pathways to determine the endocytic pathways contributed to lipoplexes gene delivery. The results indicated that transfection efficiency was decreased after incubation with inhibitors, both through the clathrin and caveolae-mediated pathways inhibition. However, inhibition of caveolin significantly reduced the uptake of lipoplexes. It was suggested that the mechanism of cationic liposomes mediated transmembrane gene delivery mainly dependent caveolae-mediated pathway. Taken together, these results demonstrated that uptake of lipoplexes in HEp-2 cells was mediated via both the clathrin-mediated and caveolae-mediated pathway. It is not definite which is the main. These results are of importance for successful gene transport via clathrin-mediated and caveolae-mediated uptake and rational design of cationic lipid gene delivery sys- tems.

\section{Acknowledgements}

The study was supported by the National Natural Science Foundation of China (21046008 and 21176046) and the Fundamental Research Funds for the Central Universities (DC120101042).

\section{REFERENCES}

[1] K. A. High. Gene therapy-the moving finger. Nature 435: 577-579, 2005.

[2] Y. Zhang, H. M. Li and S. Jing, DC-Chol/DOPE cationic liposomes: A comparative study of the influence factors on plasmid pDNA and siRNA gene delivery. International Journal of Pharmaceutics May 10;390(2):198-207, 2010.

[3] J. Rejman, A. Bragonzi and M. Conese, Role of clathrin- and caveolae mediated endocytosis in gene transfer mediated by lipoand polyplexes. Mol Ther 2005;12:468-74,2005.

[4] M. Satyajit and E. P. Richard, Pathways of clathrin-independent endocytosis. Nat Rev 8:603-12,2007.

[5] K. Sandvig , S. Pust and T. Skotland, Clathrin-independent endocytosis: mechanisms and function. Curr Opin Cell Biol.Aug;23(4):413-20, 2011.

[6] M. T. Howes, S. Mayor and R. G. Parton, Molecules, mechanisms, and cellular roles of clathrin-independent endocytosis. Curr Opin Cell Biol 22(4): 519-27, 2010.

[7] B. Emmanuelle, B. Sandrine and G. Lucie, Hepatitis C Virus Entry Depends on Clathrin-Mediated Endocytosis. J Virol. 80(14):6964-6972, July 2006.

[8] D. Hoekstra, J. Rejman and L. Wasungu, Gene delivery by cationic lipids: in and out of an endosome.Biochem.Soc Trans. Feb;35(Pt 1):68-71,2007.

[9] Y. Obata, G. Ciofani and V. Raffa, Evaluation of cationic liposomes composed of an amino acid-based of an endosome. Biochemical Society Transactions 35: 68-71,2010. 